\title{
Sniff nasal inspiratory pressure in the longitudinal assessment of young Duchenne muscular dystrophy children
}

\author{
Véronique Nève ${ }^{1,2}$, Jean-Marie Cuisset ${ }^{3}$, Jean-Louis Edmé ${ }^{1,2}$, Alain Carpentier $^{4}$, \\ Mike Howsam ${ }^{2,5}$, Olivier Leclerc ${ }^{1}$ and Régis Matran ${ }^{1,2}$
}

Affiliations: 'Service d'Explorations Fonctionnelles Respiratoires, CHRU de Lille, Lille, ${ }^{2}$ Univ Lille Nord de France, UDSL, Lille, ${ }^{3}$ Service de Neurologie Pédiatrique, CHRU de Lille, Lille, ${ }^{4}$ Centre Marc Sautelet, Villeneuve d'Ascq, and ${ }^{5}$ Centre Universitaire de Mesure et d'Analyse, Faculté de Pharmacie, Université de Lille 2, Lille, France.

Correspondence: V. Nève, Service d'Explorations Fonctionnelles Respiratoires, Hôpital Calmette, CHRU de Lille, 2, Avenue Oscar Lambret, 59000 Lille, France. E-mail: veronique.nevedachru-lille.fr

ABSTRACT Traditional measures of respiratory function in children with Duchenne muscular dystrophy (DMD) are based on maximal inspiratory pressure (PImax) and vital capacity (VC). Sniff nasal inspiratory pressure (SNIP) measurements are easily performed by young children with neuromuscular disorders. The clinical value of SNIP in the longitudinal assessment of respiratory weakness remains to be assessed. The objective of the present study was to assess longitudinally the changes in SNIP, PImax and VC with age in DMD children. We hypothesised that their longitudinal assessment would show an earlier decline in SNIP than VC.

A 3-year, prospective follow-up, at 6-month intervals of, 33 steroid-naïve, 5-20-year-old DMD patients was analysed using a linear mixed model.

SNIP measurements were reliable (within-session coefficient of variation $8 \%$ ). SNIP and VC increased until 10.5 and 12.5 years of age, respectively, and declined thereafter, while PImax did not change with age.

SNIP was an earlier marker of decline in respiratory muscle strength (at 10.5 years) than VC (at 12.5 years) in young DMD patients. SNIP longitudinal assessment is useful in the detection of inspiratory strength decline in young DMD patients when VC values remain within normal values and as an outcome measure in clinical trials for emerging therapeutics in young DMD patients from the age of 5 years.

@ERSpublications

Earlier sniff nasal inspiratory pressure than vital capacity decline in follow-up of Duchenne muscular dystrophy children http://ow.ly/mn24K

This article has supplementary material available from www.erj.ersjournals.com

Received: Aug 132012 | Accepted after revision: Dec 12 2012 | First published online: Dec 202012

Support statement: The research was supported by a grant from the French Ministry of Health (PHRC number 2001 1937).

Conflict of interest: None declared.

Copyright (OERS 2013 


\section{Introduction}

Duchenne muscular dystrophy (DMD) and Becker muscular dystrophy (BMD), the most common neuromuscular diseases in children, occur as a result of mutations in the dystrophin gene, with BMD having a milder phenotypic expression [1]. Respiratory impairment in neuromuscular diseases is assessed by the measurement of maximal inspiratory pressure (PImax), slow vital capacity (VC) and, more recently, sniff nasal inspiratory pressure (SNIP). Studies describing longitudinal follow-up of respiratory function for $\geqslant 2$ years in children with BMD or DMD have been based on PImax measurements [2-4] and lung volumes [2-7]. At the time of writing, there was no published prospective study describing longitudinal assessment of inspiratory muscle strength by SNIP measurements. SNIP has the advantage of measuring inspiratory pressure during a natural manoeuvre that is easily performed even by young children with neuromuscular disorders $[8,9]$ and may prove to be more reliable in BMD/DMD children than PImax. Moreover, based on the shape of the normal pressure-volume curve, a loss of respiratory muscle strength is expected before a fall in VC and other lung volumes [10]. Therefore, SNIP measurement may detect the respiratory muscle strength decrease earlier in the disease or in younger children than PImax or VC, and could prove to be a more sensitive outcome measurement specifically evaluating inspiratory muscle strength. Therefore, the aim of the study was to assess inspiratory pressure and volume change with age in BMD and non-steroid treated DMD children in order to: 1) document the natural evolution of SNIP measurement in these phenotypes, and 2) identify the ages of decline in SNIP, PImax and VC in DMD children by using a statistical analysis adapted to longitudinal data. Our hypothesis was that the longitudinal assessment of these parameters would show an earlier decline in SNIP than VC in DMD children.

\section{Subjects and methods}

Subjects

Twice-yearly evaluation of lung volume and inspiratory muscle strength over a period of 3 years was proposed to $51 \mathrm{BMD} / \mathrm{DMD}$ children by their neuropaediatrician between June 2002 and April 2006. Included children had BMD/DMD confirmed by muscle biopsy (dystrophin of altered size and/or abundance for BMD, and absence of dystrophin for DMD), an age $\geqslant 3$ years and all were able to perform sniff manoeuvres adequate for SNIP measurement. One BMD patient refused to participate. Four BMD and three DMD patients unable to perform acceptable sniff manoeuvres were excluded. 43 children, 10 BMD (all ambulatory at inclusion) and $33 \mathrm{DMD}$ (none treated with steroids, 13 ambulatory at inclusion), were included. Confirmation of diagnosis by genetic testing for a dystrophin gene mutation was obtained in nine BMD and 32 DMD cases.

Pulmonary function tests (PFTs) and arterialised capillary blood gas analyses (online supplementary material), none performed within 8 weeks following a respiratory tract infection, were obtained from June 2002 to May 2009. The study was approved by the ethical committee of our institution (Comité Consultatif de Protection des Personnes se Prêtant à la Recherche Biomédicale du CHRU de Lille, Lille, France) on March 6, 2001. All parents and patients signed a consent form for participation in the study.

\section{Assessments}

Height was measured, or estimated from arm span (from fingertip to fingertip) in patients with kyphoscoliosis [11].

SNIP was measured in an occluded nostril during a maximal sniff through the contralateral nostril; PImax was measured against an obstructed mouthpiece with a small leak to prevent glottic closure, at functional residual capacity (FRC), maintaining maximal pressure for $\geqslant 1 \mathrm{~s}$, and slow VC was measured according to standard procedures [12] using Hyp Air Compact (Medisoft, Dinant, Belgium). At least 10 (most often, 15-20) maximal sniffs, performed from FRC [13], and at least five PImax measurements were obtained. The highest SNIP (from a sniff $<500 \mathrm{~ms}$ in duration) and PImax were used. Baseline results were expressed as z-scores (online supplementary material).

\section{Statistics}

Median (interquartile range or range) are presented for continuous variables and percentages for categorical variables. At inclusion, for PImax and SNIP, the within-session repeatability was evaluated by two indices: coefficient of variation $(\mathrm{CoV})$, the index of repeatability that is most frequently used in PFTs; and intraclass correlation coefficient (ICC), which has the advantage over CoV of being adjusted for the effects of the scale of measurements and, for $\mathrm{VC}$, by the difference between the largest and the next-largest manoeuvre. Median values were compared by a paired or unpaired Wilcoxon test, the correlation between SNIP/PImax ratio and VC was assessed using a Spearman's correlation test. Analysis of longitudinal data was performed using a linear mixed model (LMM) [14]. 
TABLE 1 Baseline data of the 43 children included in the study

\begin{tabular}{|c|c|c|c|}
\hline & BMD $^{\#}$ & DMD & $\begin{array}{c}\text { Wilcoxon } \\
\text { p-value }\end{array}$ \\
\hline Age years & $12.5(5.4-16.4)$ & $11.0(5.0-16.7)$ & 0.1500 \\
\hline Height $\mathrm{cm}$ & $149(116-181)$ & $136(103-170)$ & 0.0430 \\
\hline Weight kg & $43.8(22.0-93.0)$ & $29.5(17.0-80)$ & 0.0540 \\
\hline $\mathrm{BMI} \mathrm{kg} \cdot \mathrm{m}^{-2}$ & $20.1(15.2-33.7)$ & $16.5(12.5-33.8)$ & 0.0540 \\
\hline BMI z-score ${ }^{+}$ & $0.2(-0.7-4.8)$ & $-0.1(-4.8-6.3)$ & 0.2200 \\
\hline \multicolumn{4}{|l|}{ Raw data } \\
\hline SNIP $\mathrm{cmH}_{2} \mathrm{O}$ & $72(45-148)$ & $48(22-80)$ & 0.0003 \\
\hline PImax $\mathrm{cmH}_{2} \mathrm{O}$ & $76(44-146)$ & $38(17-75)$ & 0.0008 \\
\hline Slow VC L & $2.62(1.45-5.36)$ & $1.70(0.77-2.11)$ & $<0.0001$ \\
\hline SNIP/PImax & $1.08(0.59-1.71)$ & $1.17(0.65-2.35)$ & 0.2440 \\
\hline \multicolumn{4}{|l|}{ Z-scores } \\
\hline SNIP & $-1.40(-3.26-1.05)$ & $-2.20(-4.24--0.91)$ & 0.0055 \\
\hline PImax & $-0.91(-3.91-1.57)$ & $-2.63(-5.27--0.28)$ & 0.0178 \\
\hline VC & $-1.19(-1.81-0.00)$ & $-2.20(-6.25-1.23)$ & 0.0259 \\
\hline
\end{tabular}

Data are presented as median (range), unless otherwise stated. Values are expressed as raw values, or as z-scores i.e. ((recorded-predicted)/ residual standard deviation from regression line of reference values). BMD: Becker muscular dystrophy; DMD: Duchenne muscular dystrophy; BMI: body mass index; SNIP: sniff nasal inspiratory pressure; PImax: maximal inspiratory pressure; VC: vital capacity. ${ }^{\#}: \mathrm{n}=10 ;{ }^{\circledR}: \mathrm{n}=33$;

${ }^{+}$: calculated according to [15].

\section{Results \\ BMD characteristics}

Anthropometric data of our population are detailed in table 1. Genetic testing, obtained in nine of the 10 BMD patients, showed that six of them had a deletion within central rod domain II: five patients had deletions around exons 45-53, generally associated with typical BMD [16], and were all ambulatory at last visit (median age 14 years, range 12-17 years), while one patient had a deletion in the proximal portion of this domain (exons 13-41) (patient 29: 16 years at inclusion, with cramps and myalgia while walking small distances and falls as chief complaints, though remaining ambulatory at the end of the follow-up). In three BMD children from the same family (patients 34-36), a deletion in domain I (exons 3-7 of the dystrophin gene) was detected, which has been previously associated with a more severe phenotype [16]; progression was more severe for patient 34 , with loss of ambulation at 16 years of age.

\section{DMD characteristics}

Ambulation was lost at a median age of 9.4 years (interquartile range $8.3-10.4$ years). Scoliosis developed in $61 \%$ of DMD patients during the follow-up: Cobb angle remained $<30^{\circ}$ in $30 \%$ of patients, ranged from $30^{\circ}$ to $50^{\circ}$ in $30 \%$ and was $>50^{\circ}$ in $1 \%$. Spine fusion was performed in $36 \%$ of the nonambulatory patients with spinal curvature $>30^{\circ}[17,18]$ at a median age of 13.2 years (interquartile range 12.5-13.8 years). Nocturnal noninvasive ventilation (NIV) had been initiated in one subject before the beginning of the study. Follow-up until June 2012 showed that NIV was initiated in 10 subjects before the age of 17 years (the mean age for the development of overnight hypercapnia requiring night NIV in a larger DMD population [19]) and in 13 patients after 17 years of age.

\section{Data at inclusion}

SNIP and VC measurements were obtained in $100 \%$ of the children and PImax in $88 \%$ (inclusion criteria). In the BMD group, SNIP, PImax and VC were abnormal ( $<2 \mathrm{z}$-scores) in two, four and none of the patients, respectively. In the DMD group, SNIP, PImax and VC were abnormal in 21, 23 and 17 patients, respectively. Pressure and volume measurements were lower in the DMD group compared with the BMD group (table 1), while ages were comparable. SNIP/PImax ratios values were comparable in both the BMD and DMD groups, with values $\geqslant 1$ in $68 \%$ of patients, but were not correlated with VC z-scores (Spearman $r=0.087, p=0.605$ ). Repeatability of baseline measurements is reported in table 2: the within-session reproducibility was significantly better for SNIP than for PImax.

\section{Longitudinal data}

The phenotype (BMD versus DMD) did not influence the evolution of SNIP/PImax with age $(\mathrm{p}=0.904)$. A linear decrease was significant for SNIP/PImax evolution with age and SNIP/PImax was $>1$ in all children up to 10 years of age (fig. 1 ). 
TABLE 2 Repeatability of baseline measurements of the 43 children included in the study

Children in whom the parameter was measured $\mathrm{n}(\%)$
Repeatability

$\operatorname{ICC}(95 \% \mathrm{Cl})$

\section{Criterion Median (interquartile range)}

\begin{tabular}{lcccc}
\hline SNIP & $43(100)$ & $\operatorname{CoV} \%$ & $8^{\#}(6-11)$ & $0.96(0.92-0.98)$ \\
PImax & $38(88)$ & $\operatorname{CoV} \%$ & $10^{\#}(6-20)$ & $0.90(0.83-0.95)$ \\
Slow VC & $43(100)$ & $\Delta \mathrm{mL}$ & $75(40-151)$ & $0.99(0.98-0.99)$ \\
& & $\Delta \%$ & $5(2-9)$ &
\end{tabular}

ICC: interclass correlation coefficient; SNIP: sniff nasal inspiratory pressure; PImax: maximal inspiratory pressure; VC: vital capacity; CoV: coefficient of variation; $\Delta$ : difference between the two best measurements obtained in each child lexpressed in millilitres or percentage of the best value). " : within-session reproducibility evaluated by the CoV was significantly better for SNIP than for PImax ( $p=0.013)$.

In contrast, a significant effect of phenotype on age-related changes in SNIP $(p=0.002), \operatorname{PImax}(p<0.001)$ and VC $(\mathrm{p}<0.001)$ was observed. These results are therefore described separately for BMD and DMD.

\section{BMD data}

SNIP, PImax and VC increased with age (figs 2-4). Reduced inspiratory pressures during follow-up (figs 2 and 3) were noted only for patient 29.

\section{DMD data}

SNIP and VC increased until 10.5 and 12.5 years, respectively, and then decreased (figs 2 and 4). Lower SNIP and VC values were observed compared with the BMD group from the age of 6.2 and 9.1 years, respectively.

While a PImax increase with age was observed in BMD patients, LMM analysis showed no change in PImax with age in DMD; compared with the BMD group, lower PImax values were thus observed in the DMD group from the age of 9.5 years (fig. 3).

DMD children with NIV initiated before the age of 17 years exhibited a lower SNIP and a lower VC from the age of 11.5 and 13.0 years, respectively (fig. 5). In contrast, age-related changes in PImax were not related to the need for NIV prior to 17 years of age.

\section{Discussion}

This study is first to document the natural evolution of SNIP in non-steroid treated DMD and in BMD children. It shows that SNIP measurements are repeatable (table 2) and that the individual age-related SNIP

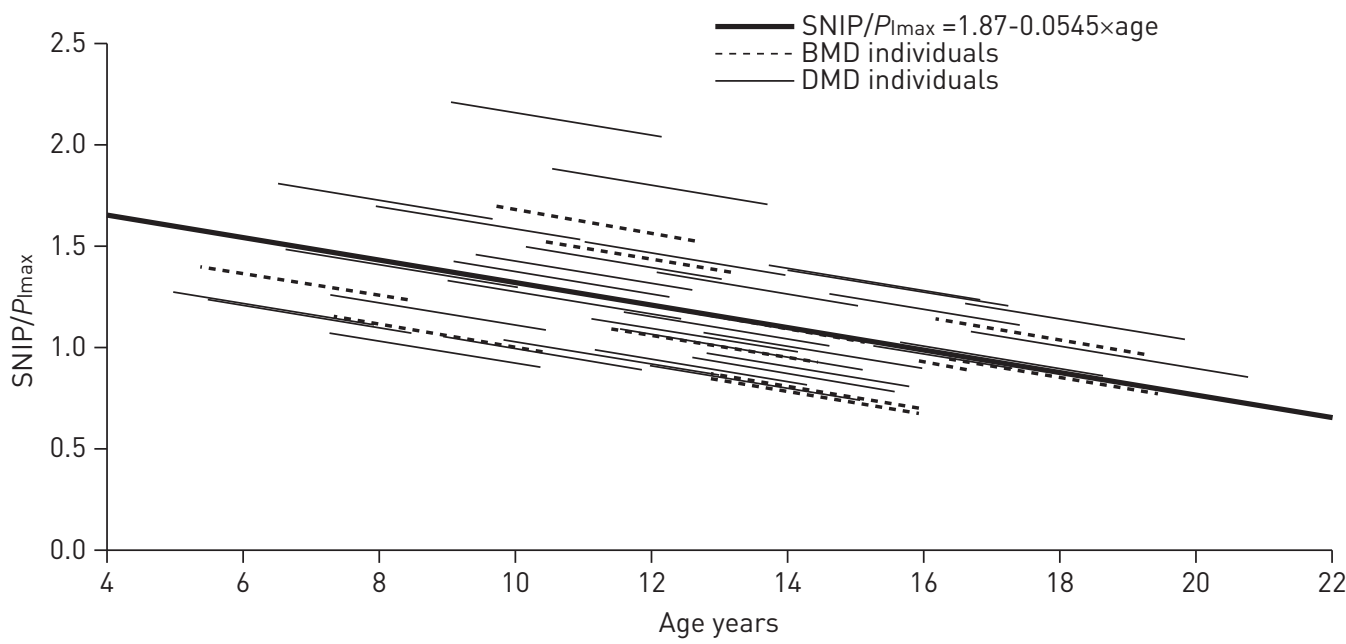

FIGURE 1 Plot of the regression line for the whole population (thick line) and individual regression lines resulting from the mixed model analysis using the sniff nasal inspiratory pressure (SNIP)/maximal inspiratory pressure (PImax) ratio values for the 10 Becker muscular dystrophy (BMD) and the 33 Duchenne muscular dystrophy (DMD) individuals. Longitudinal assessment showed that SNIP/PImax decreased with age. A linear effect was significant for the evolution of SNIP/PImax with age $(\mathrm{p}<0.001)$. The dystrophinopathy phenotype (BMD versus DMD) did not influence the evolution of SNIP/PImax with age $(p=0.904)$. SNIP/PImax was $>1$ in all children up to 10 years of age. 


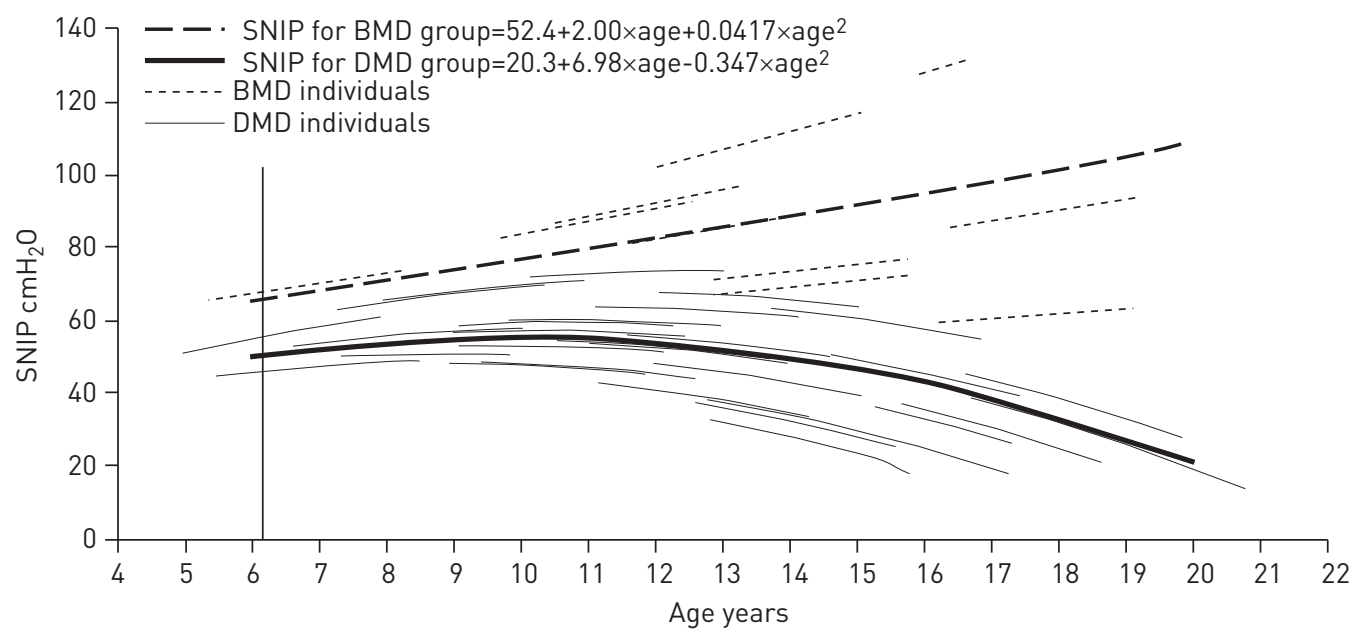

FIGURE 2 Plot of the regression line (thick lines) for each dystrophinopathy phenotype and individual regression lines (thin lines) resulting from the mixed model analysis using the 257 sniff nasal inspiratory pressure (SNIP) values for the 10 Becker muscular dystrophy (BMD) and the 33 Duchenne muscular dystrophy (DMD) individuals. A significant effect of the dystrophinopathy phenotype on age-related SNIP changes was observed $(p=0.002)$. Longitudinal assessment in children with BMD showed that SNIP increased with age. A curvilinear effect was significant for SNIP evolution with age. In DMD patients, the graphic analysis of the longitudinal individual profiles of SNIP showed an increase until 10.5 years, reaching a pressure of $55 \mathrm{cmH}_{2} \mathrm{O}$, followed by a decline with age. This curvilinearity was statistically confirmed $(\mathrm{p}=0.039)$. According to the mathematical model, compared with the BMD group, significantly lower SNIP values were observed in the DMD group from the age of 6.2 years (vertical line, $\mathrm{p}<0.050$ ).

changes reflect the severity of the DMD subphenotype. It provides more accurate assessment (online supplementary material) of age-related PImax and VC changes in both phenotypes of this dystrophinopathy and in younger children (5-year-olds) than previous studies. It emphasises the differences in age-related pressure and volume changes between BMD and DMD phenotypes. Using a statistical method that is adapted to our longitudinal study design, we identified the ages of inspiratory pressure and volume decline in DMD children. We show the following. 1) In BMD, SNIP, PImax and VC increase up to 20 years of age. In DMD, SNIP and VC increase until 10.5 and 12.5 years, and decline thereafter, and no age-related PImax change is observed. 2) The age-related pressure and volume changes therefore differ between BMD and DMD. Compared with BMD, lower pressure and volume is observed in DMD from the age of 6.2 to

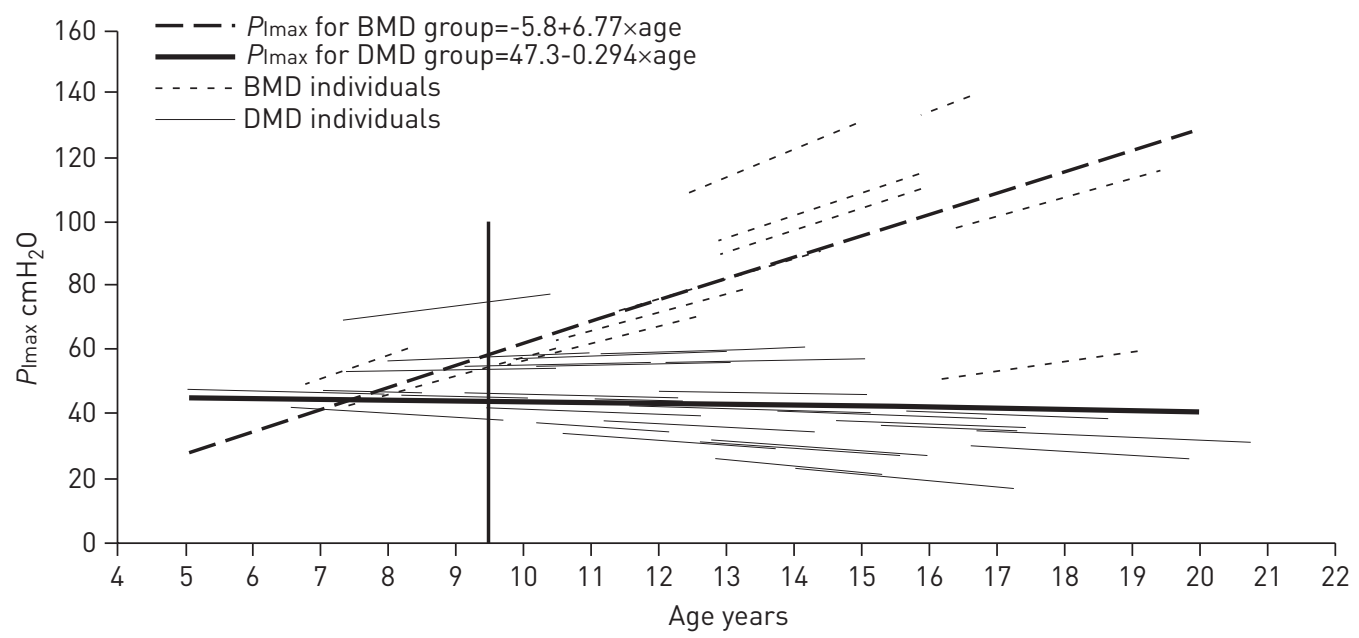

FIGURE 3 Plot of the regression line (thick lines) for each dystrophinopathy phenotype and individual regression lines resulting from the mixed model analysis using the 257 maximal inspiratory pressure (PImax) values for the 10 Becker muscular dystrophy (BMD) and the 33 Duchenne muscular dystrophy (DMD) individuals. A significant effect of the dystrophinopathy phenotype on age-related PImax changes was observed $(p<0.001)$. Longitudinal assessment in children with BMD showed that PImax increased with age. A linear effect was significant for the evolution of PImax with age. In children with DMD, the graphical analysis of the longitudinal individual profiles of PImax showed no relationship with age from 5 to 20 years $(\mathrm{p}=0.665)$. According to the mathematical model generated, compared with the BMD group, significantly lower PImax values were observed in the DMD group from 9.5 years of age (vertical line, $\mathrm{p}<0.050$ ). 


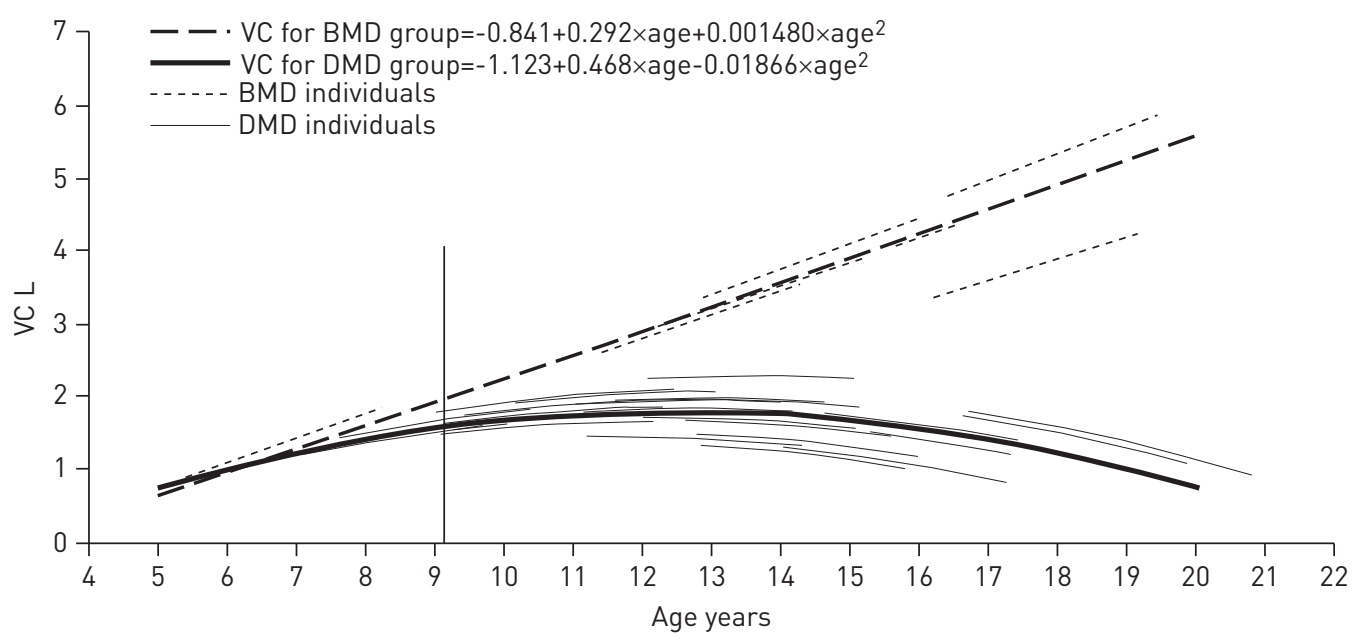

FIGURE 4 Plot of the regression line (thick lines) for each dystrophinopathy phenotype and individual regression lines resulting from the mixed model analysis using the 270 slow vital capacity (VC) values for the 10 Becker muscular dystrophy (BMD) and 33 Duchenne muscular dystrophy (DMD) individuals. A significant effect of the dystrophinopathy phenotype on VC $(p<0.001)$ was observed. Longitudinal assessment of VC in children with BMD showed that VC values increased from 5 to 20 years of age. In children with DMD, an increase in VC was observed until 12.5 years, reaching a volume of $1.82 \mathrm{~L}$, followed by a decrease in these measurements. According to the mathematical model, compared with the BMD group, significant lower VC values were observed in the DMD group from the age of 9.1 years (vertical line, $\mathrm{p}<0.05$ ).

9.5 years, with lower SNIP values being observed from an earlier age (6.2 years) than PImax (9.5 years) or VC (9.1 years). The SNIP decline was therefore an earlier sign of the decline of inspiratory muscle strength in DMD than the decline of slow VC or PImax.

\section{Baseline data}

Our data support the use of SNIP to assess inspiratory muscle strength in young children with neuromuscular disease. Many children with neuromuscular disease find it easier to perform a sniffing $[8,9]$ than a static manoeuvre [9] and our study found that BDM/DMD children exhibit a higher within-session reproducibility in performing SNIP (CoV 8\%) than PImax manoeuvres. The SNIP and PImax ICC values (higher for SNIP than PImax measurements) were consistent with those of CoV. The cognitive impairment that may be present in DMD may explain the higher variability in performing PImax manoeuvres [20], while the excellent SNIP repeatability in our study may be ascribed to the fact that sniff manoeuvres were performed with a visual feedback and that $\geqslant 10$ manoeuvres were performed. Indeed, a plateau in pressure is reached after five to 10 sniffs in most individuals and a more repeatable measure of maximum SNIP is obtained with $>10$ sniffs [13]. We therefore adopted this approach in our study, in line with the recommendations of LOFASO et al. [13] for the longitudinal monitoring of inspiratory muscle strength. As a result, the SNIP repeatability in our BMD/DMD children was better than that observed in healthy children (16-17\%, 10 sniffs without visual feedback) [21] or in 5-50-year-old subjects with neuromuscular disorders (20\%, 10 sniffs without visual feedback) [9] but close to that reported in healthy adults ( $6 \%$ and $10 \%$ with 10 and 12 sniffs, respectively, without visual feed-back) $[22,23]$.

Moreover, at inclusion, SNIP seemed to accurately reflect inspiratory muscle strength, as it was equal to or greater than PImax in $68 \%$ of our BMD/DMD patients, a proportion similar to that observed in children with neuromuscular disorders [9] but higher than that observed in an adult neuromuscular population (49\%) [24]. SNIP/PImax at inclusion was not related to VC and our results for BMD/DMD children are therefore inconsistent with those of HART et al. [24] but only two (6\%) of our DMD children had VC $<40 \%$ predicted as compared with $37 \%$ of their adult subjects.

\section{BMD longitudinal data}

SNIP and PImax

Our study has the originality of documenting the natural evolution of SNIP in BMD patients. It was interesting to obtain the same PFT assessments, including SNIP and PImax measurements, with the same schedule, in children with BMD and in children with the same dystrophinopathy but a more severe clinical phenotypic expression (DMD). In BMD, it showed that SNIP, like PImax, increases until 20 years of age. However, in one patient with a more severe clinical BMD subphenotype, we observed an early decline in SNIP and PImax suggesting that these parameters should be evaluated in such patients. 

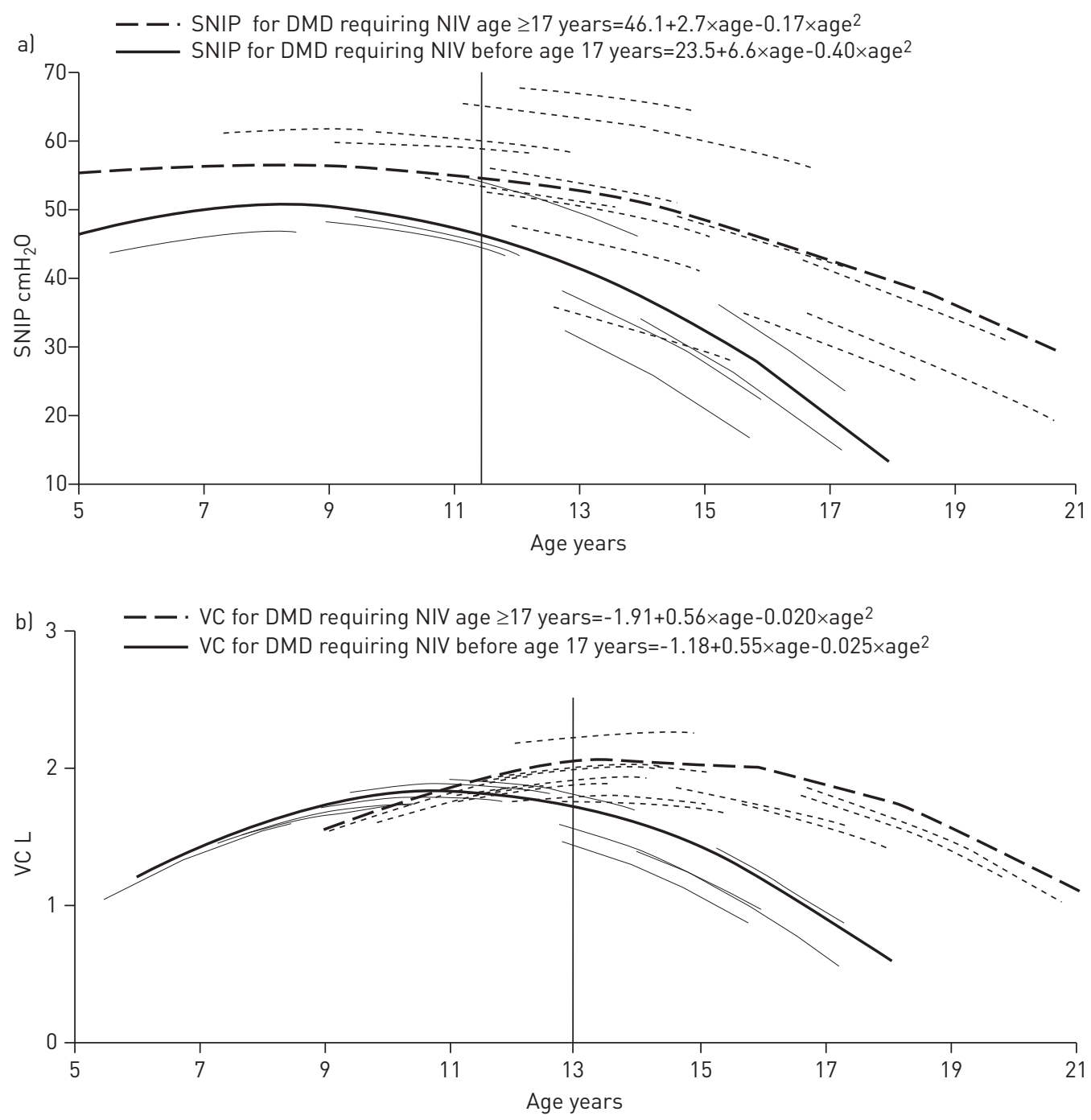

FIGURE 5 Plot of the regression lines (thick lines) for 23 Duchenne muscular dystrophy (DMD) patients according to the initiation of noninvasive ventilation (NIV) before or after the age of 17 years and individual regression lines resulting from the mixed model analysis using a) sniff nasal inspiratory pressure (SNIP) values and b) vital capacity (VC) values. DMD children with NIV before the age of 17 years exhibited a lower SNIP from the age of 11.5 years (vertical line) and a lower VC from the age of 13 years (both $\mathrm{p}<0.05$ ).

\section{Vital capacity}

VC does not seem to be able to detect more severe clinical BMD subphenotypes, as an increase in slow VC was observed until 20 years of age in all our BMD patients (including patient 29), in agreement with the data of MCDONALD et al. [3].

\section{DMD longitudinal data}

Sniff inspiratory pressure

The sniff manoeuvre has proved to be valuable in assessing diaphragm strength using transdiaphragmatic pressure (Pdi) [25] and weakness of the diaphragm was detected by measurement of sniff Pdi in $\sim 13$-yearold DMD subjects (cross-sectional evaluation). SNIP was thus proposed as a noninvasive test of inspiratory muscle strength [26]. Our longitudinal study is the first to document the natural evolution of SNIP in steroid-naïve DMD children. In addition, it showed that, in DMD, the decline of inspiratory muscle strength evaluated with SNIP occurred earlier (at 10.5 years) than the VC decline (12.5 years). Moreover, comparing the age-related pressure and volume changes in DMD to those observed in the same dystrophinopathy but a milder phenotypic expression (i.e. BMD), it showed lower SNIP and VC in DMD from the ages of 6.2 and 9.1 years, respectively. It appears that the longitudinal assessment of SNIP, which is a direct indicator of respiratory muscle function, was the earliest marker of inspiratory muscle strength 
decline in our young DMD children. SNIP measurement, obtainable from DMD subjects as young as 5 years, could be suitable as a sensitive secondary outcome measure specifically evaluating respiratory muscle strength in children up to 10 years of age. SNIP may also provide information regarding the severity of the clinical DMD subphenotype, as children requiring earlier NIV exhibit an earlier decrease in SNIP (fig. 5).

\section{Maximal inspiratory pressure}

This study included DMD children from a younger age (5 years) than previous studies [4, 7]. Our PImax results, showing no significant evolution of PImax from 5 to 20 years of age, are in agreement with another longitudinal study [5] but differ from a transverse study showing a PImax increase from 7 to 14 years of age followed by a decrease in 15-18-year-old DMD patients [27].

\section{Vital capacity}

Our study showed that the VC of DMD children increases until 12.5 years of age (reaching maximum predicted value of $1.82 \mathrm{~L}$ ) and then decreases. These results are consistent with those of studies evaluating the age-related change in VC in DMD patients longitudinally $[5,6]$ but again differ from those of the transverse study by HAHN et al. [27]: the higher VC peak $(2.66 \mathrm{~L}, \mathrm{SD} 0.87 \mathrm{~L})$ in their 13-14-year-old DMD patients could be explained by a more favourable DMD subphenotype (data concerning ages at loss of ambulation in that population were not provided). The cross-sectional design of the study by HaHN et al. [27] may also have decreased the accuracy of assessment of individual pattern of age-related changes of pulmonary function of DMD patients [28, 29].

\section{Strengths of our study}

Due to our longitudinal study design and the use of a statistic analysis adapted to the longitudinal assessment of data (online supplementary material), this study was able to accurately describe the agerelated pressure and volume changes in DMD children.

\section{Respiratory muscle tests compared with the gold standard VC}

Presently, respiratory function in patients with DMD is monitored by routine measurement of VC [18], an indicator of both respiratory muscle function and lung and chest wall compliance. SNIP measurement should be used in young patients with neuromuscular disorders for detecting respiratory weakness. Firstly, most children from the age of 5 years are able to perform adequate SNIP tests whereas a few children with neuromuscular disorders are unable to perform acceptable and repeatable VC manoeuvres [8]. Secondly, our study showed that the SNIP measurement detects the respiratory muscle weakness of DMD children earlier than VC. Finally, comparing the age-related change in SNIP and VC in DMD patients to those of BMD patients, it showed that low SNIP values are observed at an earlier age than low VC values in the DMD group. In patients with advanced restrictive ventilatory defect secondary to neuromuscular disease, VC has, however, been described as a more sensitive marker of disease progression than maximum pressures [30].

\section{Limitations of the study}

We did not evaluate the expiratory muscle strength that also contributes to the VC but maximal expiratory pressure measurements cannot usually be obtained in subjects $<7$ years of age [30]. It would have been interesting to see whether this differs in BMD versus normal subjects, and how much difference there is between healthy controls and DMD patients, but we considered it infeasible to conduct such a 3-year study in healthy children.

\section{Conclusion}

In conclusion, the design of our study allowed accurate assessment of the change in three parameters of pulmonary function over 3 years in BMD and DMD patients. It emphasised the age-related pressure and volume differences between these conditions, i.e. increases in SNIP, PImax and VC from 5 to 20 years of age in BMD versus a SNIP decline from the age of 10.5 years, no change in PImax and a VC decline from 12.5 years in DMD. In particular, significantly different age-related changes are shown in DMD compared with BMD, with SNIP values being lower from an earlier age than PImax or VC. However, the SNIP and PImax tests are complementary [24] and should be used in combination with VC for a complete sequential assessment of inspiratory muscle strength in patients with neuromuscular disease. Our study shows that SNIP is superior to PImax in children up to 10 years of age and that SNIP is much more feasible and more repeatable than PImax, and it suggests that SNIP is useful in the detection of inspiratory strength decline in young DMD cases when VC values remain within normal values. Baseline PFTs should be performed early in the course of the disease, between 4 and 6 years of age (American Thoracic Society consensus statement 
[18]) and sitting FVC should be obtained in ambulatory DMD children $\geqslant 6$ years of age [31]. Therefore, in clinical care, we propose: 1) that longitudinal assessment of SNIP should be initiated early in the course of the disease, from 5 years of age; and 2) when a SNIP decline is observed, that additional measures of pulmonary function (including VC) and gas exchange should be assessed in order to determine the need for intensified therapy. In research, as SNIP longitudinal assessment is reliable and feasible in nearly all patients $>5$ years of age and as it is able to detect the beginning of the respiratory disease, we propose to use SNIP longitudinal assessment as a physiological outcome measure in clinical trials for emerging therapeutics for young DMD patients from the age of 5 years.

\section{Acknowledgements}

The authors are grateful to the physicians, nurses, physiotherapist and members of the Centre d'Investigation Clinique of the Service d'Explorations Fonctionnelles Respiratoires du CHRU de Lille (Lille, France) who participated in the study: L. Delassus, R. Van Bulck, M.A. Moulart, C. Zak, C.M. Methlin, C. Delille, J.P. Le Cain, S. Masson, J.M. Bruandet,

B. Maisonneuve, L. Gottrand, P. Riff, J.P. Dessaint, J.L. Seynave, R. Vasseur, C. Demoutier, A. Wilquin, C. Libersa and L. Miere.

The authors are especially indebted to the parents and the children who participated in the study.

\section{References}

1 Bushby K, Finkel R, Birnkrant DJ, et al. Diagnosis and management of Duchenne muscular dystrophy, part 1: diagnosis, and pharmacological and psychosocial management. Lancet Neurol 2010; 9: 77-93.

2 Gayraud J, Ramonatxo M, Rivier F, et al. Ventilatory parameters and maximal respiratory pressure changes with age in Duchenne muscular dystrophy patients. Pediatr Pulmonol 2010; 45: 552-559.

3 McDonald CM, Abresch RT, Carter GT, et al. Profiles of neuromuscular diseases. Becker's muscular dystrophy. Am J Phys Med Rehabil 1995; 74: Suppl. 5, S93-S103.

4 McDonald CM, Abresch RT, Carter GT, et al. Profiles of neuromuscular diseases. Duchenne muscular dystrophy. Am J Phys Med Rehabil 1995; 74: Suppl. 5, S70-S92.

5 Humbertclaude V, Hamroun D, Bezzou K, et al. Motor and respiratory heterogeneity in Duchenne patients: implication for clinical trials. Eur J Paediatr Neurol 2012; 16: 149-160.

6 Phillips MF, Quinlivan RC, Edwards RH, et al. Changes in spirometry over time as a prognostic marker in patients with Duchenne muscular dystrophy. Am J Respir Crit Care Med 2001; 164: 2191-2194.

7 Steffensen BF, Lyager S, Werge B, et al. Physical capacity in non-ambulatory people with Duchenne muscular dystrophy or spinal muscular atrophy: a longitudinal study. Dev Med Child Neurol 2002; 44: 623-632.

8 Nicot F, Hart N, Forin V, et al. Respiratory muscle testing: a valuable tool for children with neuromuscular disorders. Am J Respir Crit Care Med 2006; 174: 67-74.

9 Stefanutti D, Benoist MR, Scheinmann P, et al. Usefulness of sniff nasal pressure in patients with neuromuscular or skeletal disorders. Am J Respir Crit Care Med 2000; 162: 1507-1511.

10 De Troyer A, Borenstein S, Cordier R. Analysis of lung volume restriction in patients with respiratory muscle weakness. Thorax 1980; 35: 603-610.

11 Linderholm H, Lindgren U. Prediction of spirometric values in patients with scoliosis. Acta Orthop Scand 1978; 49: 469-474.

12 Miller MR, Hankinson J, Brusasco V, et al. Standardisation of spirometry. Eur Respir J 2005; 26: 319-338.

13 Lofaso F, Nicot F, Lejaille M, et al. Sniff nasal inspiratory pressure: what is the optimal number of sniffs? Eur Respir J 2006; 27: 980-982.

14 Cnaan A, Laird NM, Slasor P. Using the general linear mixed model to analyse unbalanced repeated measures and longitudinal data. Stat Med 1997; 16: 2349-2380.

15 Cole TJ, Bellizzi MC, Flegal KM, et al. Establishing a standard definition for child overweight and obesity worldwide: international survey. BMJ 2000; 320: 1240-1243.

16 Beggs AH, Hoffman EP, Snyder JR, et al. Exploring the molecular basis for variability among patients with Becker muscular dystrophy: dystrophin gene and protein studies. Am J Hum Genet 1991; 49: 54-67.

17 Bushby K, Finkel R, Birnkrant DJ, et al. Diagnosis and management of Duchenne muscular dystrophy, part 2: implementation of multidisciplinary care. Lancet Neurol 2010; 9: 177-189.

18 Finder JD, Birnkrant D, Carl J, et al. Respiratory care of the patient with Duchenne muscular dystrophy: ATS consensus statement. Am J Respir Crit Care Med 2004; 170: 456-465.

19 Bushby K, Connor E. Clinical outcome measures for trials in Duchenne muscular dystrophy: report from International Working Group meetings. Clin Investig (Lond) 2011; 1: 1217-1235.

20 Wingeier K, Giger E, Strozzi S, et al. Neuropsychological impairments and the impact of dystrophin mutations on general cognitive functioning of patients with Duchenne muscular dystrophy. J Clin Neurosci 2011; 18: 90-95.

21 Stefanutti D, Fitting JW. Sniff nasal inspiratory pressure. Reference values in Caucasian children. Am J Respir Crit Care Med 1999; 159: 107-111.

22 Maillard JO, Burdet L, van Melle G, et al. Reproducibility of twitch mouth pressure, sniff nasal inspiratory pressure, and maximal inspiratory pressure. Eur Respir J 1998; 11: 901-905.

23 Terzi N, Corne F, Mouadil A, et al. Mouth and nasal inspiratory pressure: learning effect and reproducibility in healthy adults. Respiration 2010; 80: 379-386.

24 Hart N, Polkey MI, Sharshar T, et al. Limitations of sniff nasal pressure in patients with severe neuromuscular weakness. J Neurol Neurosurg Psychiatry 2003; 74: 1685-1687.

25 Miller JM, Moxham J, Green M. The maximal sniff in the assessment of diaphragm function in man. Clin Sci (Lond) 1985; 69: 91-96.

26 Heritier F, Rahm F, Pasche P, et al. Sniff nasal inspiratory pressure. A noninvasive assessment of inspiratory muscle strength. Am J Respir Crit Care Med 1994; 150: 1678-1683. 
27 Hahn A, Bach JR, Delaubier A, et al. Clinical implications of maximal respiratory pressure determinations for individuals with Duchenne muscular dystrophy. Arch Phys Med Rehabil 1997; 78: 1-6.

28 Edwards LJ. Modern statistical techniques for the analysis of longitudinal data in biomedical research. Pediatr Pulmonol 2000; 30: 330-344.

29 Pattishall EN, Helms RW, Strope GL. Noncomparability of cross-sectional and longitudinal estimates of lung growth in children. Pediatr Pulmonol 1989; 7: 22-28.

30 American Thoracic Society, European Respiratory Society. ATS/ERS statement on respiratory muscle testing. Am J Respir Crit Care Med 2002; 166: 518-624.

31 Birnkrant DJ, Bushby KM, Amin RS, et al. The respiratory management of patients with duchenne muscular dystrophy: a DMD care considerations working group specialty article. Pediatr Pulmonol 2010; 45: 739-748. 